\title{
THE C. NEUMANN PROBLEM AS A COMPLETELY INTEGRABLE SYSTEM ON AN ADJOINT ORBIT
}

\author{
BY
}

TUDOR RATIU

\begin{abstract}
It is shown by purely Lie algebraic methods that the C. Neumann problem-the motion of a material point on a sphere under the influence of a quadratic potential-is a completely integrable system of Euler-Poisson equations on a minimal-dimensional orbit of a semidirect product of Lie algebras.
\end{abstract}

1. The C. Neumann problem. The motion of a point on the sphere $S^{n-1}$ under the influence of a quadratic potential $U(\mathbf{x})=\frac{1}{2} A \mathbf{x} \cdot \mathbf{x}, \mathbf{x} \in \mathbf{R}^{n}, A=\operatorname{diag}\left(a_{1}, \ldots, a_{n}\right)$ is a completely integrable Hamiltonian system. For $n=3$ this has been shown by $\mathrm{C}$. Neumann in 1859 [12] and for arbitrary $n$ by K. Uhlenbeck [16], R. Devaney [3], J. Moser [10], [11], M. Adler, and P. van Moerbeke [2]. In this paper we show how this problem fits naturally in the framework of Euler-Poisson equations [4], [5], [14], [17] proving that the $C$. Neumann problem is a Hamiltonian system on a minimaldimensional adjoint orbit in a semidirect product of Lie algebras. Thus its complete integrability will follow entirely from Lie algebraic considerations.

The equations of motion are

$$
\ddot{x}_{i}=-a_{i} x_{i}+\lambda x_{i}, \quad i=1, \ldots, n,
$$

where the Lagrange multiplier $\lambda=A \mathbf{x} \cdot \mathbf{x}-\|\mathbf{x}\|^{2}$ is chosen such that $\mathbf{x} \in S^{n-1}$ during the motion. Set $\dot{\mathbf{x}}=\mathbf{y}$ and get the equivalent system to (1.1)

$$
\dot{x}_{i}=y_{i}, \quad \dot{y}_{i}=-a_{i} x_{i}+\left(A \mathbf{x} \cdot \mathbf{x}-\|\mathbf{y}\|^{2}\right) x_{i}, \quad\|\mathbf{x}\|=1, \quad \mathbf{x} \cdot \mathbf{y}=0 .
$$

The following crucial remark that motivated the present investigation is due to $K$. Uhlenbeck and can be verified without any difficulties.

Lemma 1.1. Put $X=\left(x_{i} x_{j}\right), P=\left(y_{i} x_{j}-x_{i} y_{j}\right)$. System (1.2) is equivalent to

$$
\dot{X}=[P, X], \quad \dot{P}=[X, A], \quad\|\mathbf{x}\|=1, \quad \mathbf{x} \cdot \mathbf{y}=0 .
$$

Remark that if one replaces $X$ and $A$ by $X-\mathrm{Id} / n$ and $A-(\operatorname{Tr}(A)) \operatorname{Id} / n$ respectively, where Id is the $n \times n$ identity matrix, equations (1.3) remain unchanged. From now on we shall assume that in (1.3) this change has been made so that $X, P, A \in \operatorname{sl}(n)$. The next section gives a Lie algebraic interpretation to these equations.

Received by the editors March 12, 1980.

1980 Mathematics Subject Classification. Primary 58F07, 70H05; Secondary 53C15, 17 B99.

Key words and phrases. Complete integrability, adjoint orbit, Hamiltonian system, Euler-Poisson equations, Kirillov-Kostant-Souriau symplectic structure. 
2. The equations of motion as a Hamiltonian system on an adjoint orbit. We start by recalling a few facts about the ad-semidirect product $\mathbb{S}_{\text {ad }} \times \overline{\mathbb{S}}$ of a semisimple 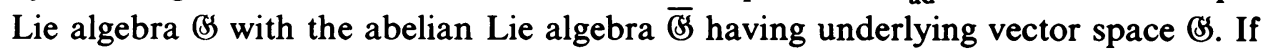 $\left(\xi_{1}, \eta_{1}\right),\left(\xi_{2}, \eta_{2}\right) \in \mathscr{S}_{\text {ad }} \times \overline{\mathscr{S}}$, their bracket is defined by

$$
\left[\left(\xi_{1}, \eta_{1}\right),\left(\xi_{2}, \eta_{2}\right)\right]=\left(\left[\xi_{1}, \xi_{2}\right],\left[\xi_{1}, \eta_{2}\right]-\left[\xi_{2}, \eta_{1}\right]\right) .
$$

If $\kappa$ denotes a bilinear, symmetric, nondegenerate, bi-invariant, two-form on $\&$, the form $\kappa_{s}$, called the semidirect product of $\kappa$ with itself and defined by

$$
\kappa_{s}\left(\left(\xi_{1}, \eta_{1}\right),\left(\xi_{2}, \eta_{2}\right)\right)=\kappa\left(\xi_{1}, \eta_{2}\right)+\kappa\left(\xi_{2}, \eta_{1}\right)
$$

is a bilinear, symmetric, nondegenerate, bi-invariant, two-form on $\mathscr{S}_{\text {ad }} \times \bar{ङ}$.

Let $G$ be a Lie group with Lie algebra (S. The Ad-semidirect product $G_{\text {Ad }} \times \overline{\mathscr{S}}$ is a Lie group with underlying manifold $G \times \mathbb{S}$ and composition law

$$
\left(g_{1}, \xi_{1}\right)\left(g_{2}, \xi_{2}\right)=\left(g_{1} g_{2}, \xi_{1}+\operatorname{Ad}_{g_{1}} \xi_{2}\right) \text {. }
$$

Note that the identity element is $(e, 0)$ and the inverse $(g, \xi)^{-1}=\left(g^{-1},-\operatorname{Ad}_{g^{-1}} \xi\right)$. The Lie algebra of $G_{A d} \times \overline{\mathscr{S}}$ is $\mathbb{S S}_{\text {ad }} \times \overline{\mathscr{S}}$. The adjoint action of the Lie group

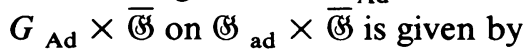

$$
\operatorname{Ad}_{(g, \theta)}(\xi, \eta)=\left(\operatorname{Ad}_{g} \xi, \operatorname{Ad}_{g} \eta+\left[\theta, \operatorname{Ad}_{g} \xi\right]\right) .
$$

In the considerations that follow, the orbit symplectic structure plays a central role (see Abraham and Marsden [1] and Ratiu [14] for proofs). If a Lie algebra (S) has a bilinear, symmetric, nondegenerate, bi-invariant two-form $\kappa$,

$$
\omega_{\xi}\left(\operatorname{Ad}_{g} \xi\right)\left(\left[\eta, \operatorname{Ad}_{g} \xi\right],\left[\zeta, \operatorname{Ad}_{g} \xi\right]\right)=-\kappa\left([\eta, \zeta], \operatorname{Ad}_{g} \xi\right)
$$

for $\xi, \eta, \zeta \in \mathbb{S}, g \in G$, defines the canonical symplectic structure on the adjoint orbit $G \cdot \xi$ through $\xi$. If $E, E^{\prime}:$ SS $\rightarrow \mathbf{R}$, the Hamiltonian vector field of $E \mid G \cdot \xi$ is given by

$$
X_{E \mid G \cdot \xi}\left(\operatorname{Ad}_{g} \xi\right)=-\left[(\operatorname{grad} E)\left(\operatorname{Ad}_{g} \xi\right), \operatorname{Ad}_{g} \xi\right]
$$

and the Poisson bracket of $E\left|G \cdot \xi, E^{\prime}\right| G \cdot \xi$ is

$$
\left\{E\left|G \cdot \xi, E^{\prime}\right| G \cdot \xi\right\}\left(\operatorname{Ad}_{g} \xi\right)=-\kappa\left(\left[(\operatorname{grad} E)\left(\operatorname{Ad}_{g} \xi\right),\left(\operatorname{grad} E^{\prime}\right)\left(\operatorname{Ad}_{g} \xi\right)\right], \operatorname{Ad}_{g} \xi\right)
$$

where grad denotes the gradient with respect to $\kappa$.

For the semidirect product these formulas become

$$
\begin{gathered}
\omega(\xi, \eta)\left(\left[(\xi, \eta),\left(\zeta_{1}, \zeta_{1}^{\prime}\right)\right],\left[(\xi, \eta),\left(\zeta_{2}, \zeta_{2}^{\prime}\right)\right]\right) \\
=-\kappa_{s}\left((\xi, \eta),\left[\left(\zeta_{1}, \zeta_{1}^{\prime}\right),\left(\zeta_{2}, \zeta_{2}^{\prime}\right)\right]\right) \\
\left.X_{E}(\xi, \eta)=-\left(\left[\operatorname{grad}_{2} E\right)(\xi, \eta), \xi\right],\left[\left(\operatorname{grad}_{2} E\right)(\xi, \eta), \eta\right]+\left[\left(\operatorname{grad}_{1} E\right)(\xi, \eta), \xi\right]\right) \\
\left\{E, E^{\prime}\right\}(\xi, \eta)=-\kappa\left(\xi,\left[\left(\operatorname{grad}_{2} E\right)(\xi, \eta),\left(\operatorname{grad}_{1} E^{\prime}\right)(\xi, \eta)\right]\right) \\
-\kappa\left(\xi,\left[\left(\operatorname{grad}_{1} E\right)(\xi, \eta),\left(\operatorname{grad}_{2} E^{\prime}\right)(\xi, \eta)\right]\right) \\
-\kappa\left(\eta,\left[\left(\operatorname{grad}_{2} E\right)(\xi, \eta),\left(\operatorname{grad}_{2} E^{\prime}\right)(\xi, \eta)\right]\right)
\end{gathered}
$$

where $\left(\operatorname{grad}_{1}, \operatorname{grad}_{2}\right)$ denotes the usual gradient with respect to $\kappa \times \kappa$; note that the gradient with respect to $\kappa_{s}$ is $\left(\operatorname{grad}_{2}, \operatorname{grad}_{1}\right)$. 
Assume that $\mathscr{S}=\mathfrak{\Re} \bigoplus \mathfrak{R}$, where $\mathfrak{R}$ is a vector subspace and $\mathfrak{R}$ a Lie subalgebra of $\mathscr{S}, \mathfrak{R}$ having $N$ as underlying closed Lie subgroup of $G$. Denote by $\Pi_{\mathfrak{R}}, \Pi_{\mathfrak{R}}$ the projections of $\mathbb{S}$ on $\Re$ and $\mathfrak{N}$ respectively. Then $\mathbb{S}^{*}=\mathfrak{\Omega}^{*} \oplus \mathfrak{R}^{*}$. The nondegeneracy of $\kappa$ on $\mathscr{S}$ defines the isomorphisms $\mathfrak{R}^{\perp} \cong \Omega^{*}, \Re^{\perp} \simeq \mathfrak{R}^{*}$ and thus the coadjoint action of $N$ on $\mathfrak{R}^{*}$ induces an action of $N$ on $\Omega^{\perp}$ given by

$$
N \times{\Omega^{\perp}}^{\perp}(n, \xi) \mapsto \Pi_{\Omega^{\perp}} \operatorname{Ad}_{n} \xi \in \Re^{\perp}
$$

where $\Pi_{\Omega^{\perp}}: \mathscr{S} \rightarrow \mathscr{\Omega}^{\perp}$ denotes the canonical projection defined by the direct sum decomposition $\mathscr{S}=\mathfrak{R}^{\perp} \oplus \mathfrak{N}^{\perp}$. Thus the orbit $N \cdot \xi$ equals

$$
N \cdot \xi=\left\{\Pi_{\Omega^{\perp}}\left(\operatorname{Ad}_{n} \xi\right) \mid n \in N\right\} \subseteq \Re^{\perp}, \quad \xi \in \Re^{\perp},
$$

whose tangent space at $\bar{\xi} \in N \cdot \xi$ is

$$
T_{\bar{\xi}}(N \cdot \xi)=\left\{\Pi_{\Re^{\perp}}[\bar{\xi}, \eta] \mid \eta \in \Re\right\} \subseteq \Re^{\perp} .
$$

The symplectic structure on $N \cdot \xi$ equals, by (2.5),

$$
\omega_{\xi}(\bar{\xi})\left(\Pi_{\Omega^{\perp}}[\eta, \bar{\xi}], \Pi_{\Re^{\perp}}[\zeta, \bar{\xi}]\right)=-\kappa([\eta, \zeta], \bar{\xi}), \quad \bar{\xi} \in N \cdot \xi \subseteq \Re^{\perp},
$$

and the Hamiltonian vector field defined by $E \mid N \cdot \xi, E:$ (S) $\rightarrow \mathbf{R}$, is, by (2.6),

$$
X_{E \mid N \cdot \xi}(\bar{\xi})=-\Pi_{\Re^{\perp}}\left[\Pi_{\mathfrak{R}}(\operatorname{grad} E)(\bar{\xi}), \bar{\xi}\right], \quad \bar{\xi} \in N \cdot \xi \subseteq \Re^{\perp} .
$$

Finally the Poisson bracket of $E\left|G \cdot \xi, E^{\prime}\right| G \cdot \xi$ is given by (2.7),

$$
\left\{E\left|G \cdot \xi, E^{\prime}\right| G \cdot \xi\right\}(\bar{\xi})=-\kappa\left(\left[\Pi_{\mathfrak{R}}(\operatorname{grad} E)(\bar{\xi}), \Pi_{\mathfrak{R}}\left(\operatorname{grad} E^{\prime}\right)(\bar{\xi})\right], \bar{\xi}\right),
$$

for $\bar{\xi} \in N \cdot \xi \subseteq \Omega^{\perp}$. All previous considerations naturally live on the duals but this is the form we shall use for the C. Neumann problem; see Ratiu [14] for a parallel description on duals.

We shall apply all previous results to a specific Lie algebra. Let $\mathbb{S}=s l(n)_{\text {ad }} \times \overline{s l(n)}, G=S l(n)_{\text {Ad }} \times \overline{s l(n)}, \mathfrak{N}=s o(n) \times s y m, N=S O(n) \times s y m$, $\mathfrak{\Re}=\operatorname{sym} \times \operatorname{so}(n)$, where $\operatorname{sym} \subset \operatorname{sl}(n)$ denotes the vector space of all symmetric matrices. Clearly $\mathfrak{N}$ is a Lie subalgebra and $\Re$ a vector subspace of $\mathbb{S}, N$ a Lie subgroup of $G$ with Lie algebra $\mathfrak{R}$. Thus by our general considerations $N$ acts on $\Omega^{\perp}$. It is easy to check that with respect to $\kappa_{s}$, where $\kappa(A, B)=-\frac{1}{2} \operatorname{Tr}(A B), \Omega^{\perp}=\Re$, $\mathfrak{R}^{\perp}=\mathfrak{R}$. In what follows we shall determine explicitly a particular $N$-orbit; note first that in the case above $\Pi_{\Omega^{\perp}}$ in formula (2.11) is not necessary, i.e. the action of $N$ on $\Omega^{\perp}$ is given by (2.4).

If $\mathbf{y}, \mathbf{z} \in \mathbf{R}^{n}$, denote by $\mathbf{y} \otimes \mathbf{z}$ the matrix having entries $y_{i} z_{j}$ and remark that if $g \in S O(n), g(\mathbf{y} \otimes \mathbf{z}) g^{-1}=(g \mathbf{y}) \otimes(g z)$. Let $\mathbf{z}=(1, \ldots, 1) / \sqrt{n}$ and take $(\mathbf{z} \otimes \mathbf{z}$ $-\mathrm{Id} / n, 0) \in \Re^{\perp}$. Let $g \in S O(n)$ be arbitrary and denote $\mathbf{x}=g \mathbf{z}$. Then $\|\mathbf{x}\|=\|\mathbf{z}\|$ $=1$ and $g(\mathbf{z} \otimes \mathbf{z}-\mathrm{Id} / n) g^{-1}=\mathbf{x} \otimes \mathbf{x}-\mathrm{Id} / n$ which is a matrix $X$ having all off-diagonal entries equal to $x_{i} x_{j}$ and diagonal entries $x_{i}^{2}-1 / n$. Thus the first component of the $N$-orbit through $(\mathrm{z} \otimes \mathrm{z}-\mathrm{Id} / n, 0)$ is the matrix $X$ occurring in Lemma 1.1. We compute the second component. If $\theta \in \operatorname{sym}, X_{i j}=x_{i} x_{j}, X_{i i}=x_{i}^{2}$ $-1 / n$, then

$$
[\theta, X]_{i j}=\left(\sum_{k=1}^{n} \theta_{i k} x_{k}-x_{i} C(\mathbf{x}, \theta)\right) x_{j}-x_{i}\left(\sum_{k=1}^{n} \theta_{j k} x_{k}-x_{j} C(\mathbf{x}, \theta)\right),
$$


where $C(\mathbf{x}, \theta)=\sum_{k, i} x_{i} x_{k} \theta_{i k}$. Put $y_{i}=\sum_{k=1}^{n}\left(\theta_{i k} x_{k}-x_{i} C(\mathbf{x}, \theta)\right)$ and remark that if $\mathbf{y}=\left(y_{1}, \ldots, y_{n}\right), \mathbf{x} \cdot \mathbf{y}=0$ since $\|\mathbf{x}\|=1$. Thus the second component of the $N$-orbit consists of matrices $P \in s o(n), P_{i j}=y_{i} x_{j}-x_{j} y_{i}, \mathbf{x} \cdot \mathbf{y}=0$. We showed hence that this $N$-orbit consists of pairs $(X, P) \in \operatorname{sym} \times \operatorname{so}(n)$ with $X, P$ defined as in Lemma 1.1.

Remark that the correspondence $(X, P)=\lambda(\mathbf{x}, \mathbf{y})$ defines a diffeomorphism of this orbit onto the tangent bundle $T S^{n-1}$ of the unit sphere $S^{n-1}$ in $\mathbf{R}^{n}$. A tangent vector at $(X, P)$ to this orbit is $[(X, P),(\xi, \eta)]$ for $(\xi, \eta) \in \operatorname{so}(n) \times \operatorname{sym}$ and is of the form $(V, W) \in \operatorname{sym} \times \operatorname{so}(n)$, where $V_{i j}=v_{j} x_{i}+x_{j} v_{i}, v_{i}=\sum_{k=1}^{n} x_{k} \xi_{k i}, W_{i j}=w_{i} x_{j}$ $-x_{i} w_{j}+y_{i} v_{j}-y_{j} v_{i}, w_{i}=\sum_{k=1}^{n}\left(y_{k} \xi_{k i}-x_{k} \eta_{k i}\right)+x_{i} \sum_{k, i} x_{i} x_{k} \eta_{i k}$ as a short calculation shows. Thus the tangent map of $\lambda$ is given by $(V, W) \mapsto(v, w)$, where $\mathbf{v}=\left(v_{1}, \ldots, v_{n}\right), \mathbf{w}=\left(w_{1}, \ldots, w_{n}\right)$.

$T S^{n-1}$ has a natural symplectic structure induced by the canonical symplectic form $-\sum_{i=1}^{n} d x_{i} \wedge d y_{i}$ of $\mathbf{R}^{2 n}$. By (2.8) the canonical symplectic structure $\omega$ on the orbit is given by

$$
\omega(X, P)\left(\left[(X, P),\left(\xi^{1}, \eta^{1}\right)\right],\left[(X, P),\left(\xi^{2}, \eta^{2}\right)\right]\right)=\kappa_{s}\left(\left[\left(\xi^{2}, \eta^{2}\right),\left(\xi^{1}, \eta^{1}\right)\right],(X, P)\right) .
$$

Let $V^{i}, W^{i}, \mathbf{v}^{i}, \mathbf{w}^{i}$ be defined by $\xi^{i}, \eta^{i}, i=1,2$. We have by bi-invariance of $\kappa_{s}$, antisymmetry of $\xi^{2}$, symmetry of $\eta^{1}$, and by (2.8), (2.14)

$$
\begin{aligned}
\left(\lambda_{*} \omega\right)(\mathbf{x}, \mathbf{y}) & \left(\left(\mathbf{v}^{1}, \mathbf{w}^{1}\right),\left(\mathbf{v}^{2}, \mathbf{w}^{2}\right)\right)=\omega(X, P)\left(\left(V^{1}, W^{1}\right),\left(V^{2}, W^{2}\right)\right) \\
& =-\kappa_{s}\left(\left(\xi^{2}, \eta^{2}\right),\left(V^{1}, W^{1}\right)\right) \\
& =\frac{1}{2} \operatorname{Tr}\left(\xi^{2} W^{1}\right)+\frac{1}{2} \operatorname{Tr}\left(\eta^{2} V^{1}\right) \\
& =\sum_{k=1}^{n}\left(w_{k}^{1} \sum_{i=1}^{n} x_{i} \xi_{i k}^{2}\right)-\sum_{k=1}^{n}\left(v_{k}^{1} \sum_{i=1}^{n} y_{i} \xi_{i k}^{2}\right)+\sum_{k=1}^{n}\left(v_{k}^{1} \sum_{i=1}^{n} x_{i} \eta_{i k}^{2}\right) \\
& =-\sum_{k=1}^{n}\left(v_{k}^{1} w_{k}^{2}-w_{k}^{1} v_{k}^{2}\right) \\
& =\left(-\sum_{k=1}^{n} d x_{k} \wedge d y_{k}\right)\left(\left(\mathbf{v}^{1}, \mathbf{w}^{1}\right),\left(\mathbf{v}^{2}, \mathbf{w}^{2}\right)\right) .
\end{aligned}
$$

This shows that $\lambda$ is a symplectic diffeomorphism:

$$
\lambda_{*} \omega=\left(-\sum_{i=1}^{n} d x_{i} \wedge d y_{i}\right) \mid T S^{n-1}
$$

Let $L: s l(n) \rightarrow s l(n)$ be given by $L(\xi)=-\xi . L$ is clearly a $\kappa$-symmetric isomorphism. The following Euler-Poisson Hamiltonian (see [4], [5], [15], [17] for motivations) $E(\xi, \eta)=\frac{1}{2} \kappa(\eta, L(\eta))+\kappa(A, \xi)$ for $A \in s l(n)$ a fixed diagonal matrix, induces a Hamiltonian vector field on the $N$-orbit through $(\mathbf{z} \otimes \mathbf{z}-\mathrm{Id} / n, 0)$ given by $(2.15),(X, P) \mapsto([P, X],[X, A])$, i.e. we get equations (1.3). Hence we proved the following. 
THEOREM 2.1. The $N=s o(n) \times$ sym-orbit through $(\mathbf{z} \otimes \mathbf{z}-\mathrm{Id} / n, 0)$ in $\AA^{\perp}=\Re$ $=\operatorname{sym} \times \operatorname{so}(n), \mathrm{z}=(1, \ldots, 1) / \sqrt{n}$, consists of all pairs $(X, P), X_{i j}=x_{i} x_{j}$ for $i \neq j, X_{i i}=x_{i}^{2}-1 / n, P_{i j}=y_{i} x_{j}-x_{i} y_{j},\|\mathbf{x}\|=1, \mathbf{x} \cdot \mathbf{y}=0$. With the KirillovKostant-Souriau symplectic structure this $(2 n-2)$-dimensional orbit is symplectically diffeomorphic via $(X, P) \mapsto(\mathbf{x}, \mathbf{y})$ to $T S^{n-1}$ with the symplectic structure induced from $\mathbf{R}^{2 n}$ by $-\sum_{i=1}^{n} d x_{i} \wedge d y_{i}$. The Hamiltonian $E(X, P)=-\frac{1}{2} \kappa(P, P)+\kappa(A, X)$ defines on this orbit the equations of motion of the $C$. Neumann problem

$$
\dot{X}=[P, X], \quad \dot{P}=[X, A], \quad\|\mathbf{x}\|=1, \quad \mathbf{x} \cdot \mathbf{y}=0 .
$$

Remark. M. Adler and P. van Moerbeke [2] have independently observed that (2.17) is a Hamiltonian system in a semidirect product.

\section{The complete set of integrals and their involution.}

Lemma 3.1. The equations $\dot{X}=[P, X], \dot{P}=[X, A],\|\mathbf{x}\|=1, \mathbf{x} \cdot \mathbf{y}=0$ are equivalent to

$$
\left(-X+P \lambda+A \lambda^{2}\right)^{\cdot}=\left[-X+P \lambda+A \lambda^{2},-P-A \lambda\right]
$$

for any parameter $\lambda$.

The proof is a straightforward verification. It follows that the functions $(1 / 2(k+1)) \operatorname{Tr}\left(-X+P \lambda+A \lambda^{2}\right)^{k+1}$ are conserved on the flow of (3.1). If $t \mapsto$ $(X(t), P(t))$ denotes the flow of (2.17), then $t \mapsto-X(t)+P(t) \lambda+A \lambda^{2}$ is the flow of (3.1) and we conclude that the coefficients of $\lambda$ in the expansion of $(1 / 2(k+1)) \operatorname{Tr}\left(-X+P \lambda+A \lambda^{2}\right)^{k+1}$ are conserved along the flow of (2.17). Let $f_{k}(X, P)$ be the coefficient of $\lambda^{2 k}$ in this expansion for $k=1, \ldots, n-1$. We shall prove in this section that all $f_{k}$ are in involution. The method of the proof follows Ratiu [13], [15] closely.

THEOREM 3.2. Let \&S be a Lie algebra with a bilinear, symmetric, nondegenerate, bi-invariant, two-form $\kappa$. Let $f, g: \mathbb{S} \rightarrow \mathbf{R}$ satisfy $[(\operatorname{grad} f)(\xi), \xi]=0,[(\operatorname{grad} g)(\xi), \xi]$ $=0$ for all $\xi \in$ (S). Denote $f_{a}(\xi, \eta)=f\left(\xi+a \eta+a^{2} \varepsilon\right), g_{b}(\xi, \eta)=g\left(\xi+b \eta+b^{2} \varepsilon\right)$ for $\varepsilon \in \mathcal{B}$ fixed and $a, b$ arbitrary parameters. Then $f_{a}, g_{b}$ Poisson commute in the bracket of $\mathbb{S S}_{\text {ad }} \times \overline{\mathbb{S}}$ defined by its symplectic decomposition in adjoint orbits.

Proof. Clearly $\left(\operatorname{grad}_{1} f_{a}\right)(\xi, \eta)=(\operatorname{grad} f)\left(\xi+a \eta+a^{2} \varepsilon\right),\left(\operatorname{grad}_{2} f_{a}\right)(\xi, \eta)=$ $a(\operatorname{grad} f)\left(\xi+a \eta+a^{2} \varepsilon\right)$ and similarly for $g_{b}$. By (2.10)

$$
\begin{aligned}
\left\{f_{a}, g_{b}\right\}(\xi, \eta)= & -\kappa\left(\left[\left(\operatorname{grad}_{1} f_{a}\right)(\xi, \eta),\left(\operatorname{grad}_{2} g_{b}\right)(\xi, \eta)\right], \xi\right) \\
& -\kappa\left(\left[\left(\operatorname{grad}_{2} f_{a}\right)(\xi, \eta),\left(\operatorname{grad}_{1} g_{b}\right)(\xi, \eta)\right], \xi\right) \\
& -\kappa\left(\left[\left(\operatorname{grad}_{2} f_{a}\right)(\xi, \eta),\left(\operatorname{grad}_{2} g_{b}\right)(\xi, \eta)\right], \eta\right) \\
= & -\kappa\left((a+b) \xi+a b \eta,\left[(\operatorname{grad} f)\left(\xi+a \eta+a^{2} \varepsilon\right),\right.\right. \\
= & \left.\left(b^{2} /(\operatorname{grad} g)\left(\xi+b \eta+b^{2} \varepsilon\right)\right]\right) \\
& +\left(a^{2} /(a-b)\right) \kappa\left(\left[\xi+a \eta+a^{2} \varepsilon,(\operatorname{grad} f)\left(\xi+a \eta+a^{2} \varepsilon\right)\right],\right. \\
& \left.(\operatorname{grad} g)\left(\xi+b \eta+b^{2} \varepsilon\right)\right) \\
& \left.(\operatorname{grad} f)\left(\xi+a \eta+a^{2} \varepsilon\right)\right)=0
\end{aligned}
$$


by hypothesis. By continuity $\left\{f_{a}, g_{b}\right\}=0$ holds also for $a=b$.

Remark. The condition $[(\operatorname{grad} f)(\xi), \xi]=0$ for all $\xi \in(S)$ is the infinitesimal version of Ad-invariance of $f$ as an easy computation shows.

THEOREM 3.3. Let $G$ be a Lie group, $N$ a closed subgroup, with Lie algebras \&S and $\mathfrak{N}$ respectively. Assume $\mathbb{S}=\Re \oplus \mathfrak{R}, \Re$ a vector subspace, $[\Re, \mathfrak{N}] \subseteq \Re$, and that $\mathbb{S}$ has a bilinear, symmetric, nondegenerate, bi-invariant, two-form $\kappa$. Assume that $f, g$ : (S) $\rightarrow \mathbf{R}$ Poisson commute on $\mathbb{S}$, i.e.

$$
\kappa([(\operatorname{grad} f)(\xi),(\operatorname{grad} g)(\xi)], \xi)=0,
$$

for all $\xi \in \mathbb{B}$. If either

(1) $\Re$ is a Lie subalgebra, or

(2) $\Pi_{\mathfrak{R}}\left[\Pi_{\Re}(\operatorname{grad} f)(\eta), \Pi_{\Re}(\operatorname{grad} g)(\eta)\right]=0$ for all $\eta \in \mathfrak{\Omega}^{\perp}$, then on any $N$-orbit in $\Omega^{\perp}$, the functions $f, g$ Poisson commute.

Proof. Let $\eta \in \Re^{\perp}$. By hypothesis and (2.16) we get

$$
\begin{aligned}
0 & =-\kappa([(\operatorname{grad} f)(\eta),(\operatorname{grad} g)(\eta)], \eta) \\
& =\{f|N \cdot \eta, g| N \cdot \eta\}(\eta)-\kappa\left(\Pi_{\Re}\left[\Pi_{\Omega}(\operatorname{grad} f)(\eta), \Pi_{\Omega}(\operatorname{grad} g)(\eta)\right], \eta\right) .
\end{aligned}
$$

The second term vanishes in either hypothesis 1 or 2 .

REMARK. Both theorems have identical versions on duals and $\kappa$ is not needed there.

These two general theorems prove the involution of the functions $f_{k}$ in the following way. In Theorem 3.2 take $\mathbb{S}=s l(n)$ and let

$$
\phi_{k}(\xi, \eta)=(1 / 2(k+1)) \operatorname{Tr}\left(-\xi+\eta \lambda_{k+1}+A \lambda_{k+1}^{2}\right)^{k+1} .
$$

Then $\left\{\phi_{k}, \phi_{l}\right\}=0$ on $s l(n)_{\text {ad }} \times \overline{s l(n)}$ for any parameters $\lambda_{k+1}, \lambda_{l+1}$, i.e. $\phi_{k+1}$ is constant on the flow defined by $\phi_{l+1}$ no matter what $\lambda_{k+1}, \lambda_{l+1}$ are, i.e. the coefficients of $\lambda_{k+1}$ in $\phi_{k+1}$ are constant on the flow defined by $\phi_{l+1}$ for all $\lambda_{l+1}$. Hence $\left\{f_{k}, \phi_{l}\right\}=0$ for all $\lambda_{l}$ and thus $\left\{f_{k}, f_{l}\right\}=0$ for any $k, l$. In Theorem 3.3 take $\mathbb{S}=\operatorname{sl}(n)_{\text {ad }} \times \overline{s l(n)}, \mathfrak{R}=\operatorname{sym} \times \operatorname{so}(n), \mathfrak{R}=\operatorname{so}(n) \times \operatorname{sym}$ and remark that $[\mathfrak{R}, \mathfrak{R}]$ $\subseteq \Re$. $f_{k}, f_{l}$ Poisson commute on \&s by what we just proved, so in order to conclude that they Poisson commute on the $N$-orbit through $(\mathrm{z} \otimes \mathrm{z}-\mathrm{Id} / n, 0)$ we have to check condition (2) of Theorem 3.3 for $\eta=(X, P) \in \Re^{\perp}=\Re$. An easy computation shows that

$$
f_{k}(X, P)=\frac{1}{2(k+1)} \operatorname{Tr}\left(-\sum_{i=0}^{k} A^{i} X A^{k-i}+\sum_{\substack{i+j+l=k-1 \\ i, j, l>0}} A^{i} P A^{j} P A^{l}\right)
$$

so that the gradient of $f_{k}$ with respect to $\kappa_{s}$ is

$$
\left(\operatorname{grad} f_{k}\right)(X, P)=\left(-\sum_{i=0}^{k-1} A^{i} P A^{k-1-j}, A^{k}\right) \in \operatorname{so}(n) \times \operatorname{sym}=\mathfrak{N}
$$

and hence $\Pi_{\Re}\left(\operatorname{grad} f_{k}\right)(X, P)=0$.

THEOREM 3.4. The functions $f_{k}, k=1, \ldots, n-1$, are constants of the motion in involution for the $C$. Neumann problem. $f_{1}=-E, E=$ energy function. 
RemarK. Equation (3.1) is Hamiltonian in the Kac-Moody extension of $s l(n)$; see Adler and van Moerbeke [2].

4. Independence. Throughout this section we assume that $A=\operatorname{diag}\left(a_{1}, \ldots, a_{n}\right)$ has all entries distinct.

Let $\mathcal{V}=\operatorname{span}\left\{X_{f_{k}}(X, P) \mid k=1, \ldots, n-1\right\}$. We have to show that generically $\operatorname{dim}(\mathfrak{V})=n-1$.

Denote by $U_{k i}$ the coefficient of $\lambda^{i}$ in the expansion of $\left(-X+P \lambda+A \lambda^{2}\right)^{k}$. From (3.3) it follows that $\left(\operatorname{grad} f_{k}\right)(X, P)=\left(-U_{k, 2 k-1}, U_{k, 2 k}\right)$, so that $\mathcal{V}=$ $\operatorname{ad}_{(X, P)} \mathbb{Q}_{0}$, where $\mathbb{Q}_{0}=\operatorname{span}\left\{\left(-U_{k, 2 k-1}, U_{k, 2 k}\right) \mid k=1, \ldots, n-1\right\}$. Since $U_{k, 2 k}=$ $A^{k}$ and $A$ has all entries distinct we conclude that $\left\{A^{k} \mid k=1, \ldots, n-1\right\}$ are linearly independent in $\operatorname{sl}(n)$ and thus $\operatorname{dim}\left(\mathscr{Q}_{0}\right)=n-1$; in particular $\operatorname{dim}(\mathscr{V}) \leqslant$ $\operatorname{dim}\left(Q_{0}\right)=n-1$ which was already obvious from the definition of $\mathfrak{V}$.

Let $Q_{j}=\operatorname{span}\left\{\left(-U_{k, 2 k-1-2 j}, U_{k, 2 k-2 j}\right) \mid k=j, \ldots, n-1\right\}$ where we make the convention that any $U_{k i}$ with $i<0$ is identical zero; thus $\operatorname{dim}\left(\mathbb{Q}_{j}\right) \leqslant n-j, j=$ $1, \ldots, n-1$. Denote $\mathscr{V}_{j}=\operatorname{ad}_{(X, P)} \mathbb{Q}_{j}, j=0,1, \ldots, n-1$, so that $\mathscr{V}=\mathscr{V}_{0}$.

LEMMA 4.1. The linear map $f_{A, P}: s l(n) \times s l(n) \rightarrow \operatorname{sl}(n) \times s l(n)$ defined by

$$
f_{A, P}(\xi, \eta)=([\eta, P]-[\xi, A],[A, \eta])
$$

is injective on all $\mathbb{Q}_{j}, j=1, \ldots, n-1$, for generic $(X, P)$.

This is a direct, but somewhat lengthy verification (see [15] for a more complicated similar proof).

LEMMA 4.2. The following relations hold for any $k=1, \ldots, n-1$ :

$$
-\left[U_{k, 2 k-j}, X\right]+\left[U_{k, 2 k-j-1}, P\right]+\left[U_{k, 2 k-j-2}, A\right]=0 \text {. }
$$

This is obvious if one notes that the expression above is the coefficient of $\lambda^{j}$ in the expansion of $\left[\left(-X+P \lambda+A \lambda^{2}\right)^{k},-X+P \lambda+A \lambda^{2}\right] \equiv 0$.

We have thus by the two prior lemmas

$$
\begin{aligned}
\operatorname{ad}_{(X, P)}(- & \left.U_{k, 2 k-1-2 j}, U_{k, 2 k-2 j}\right) \\
& =\left(\left[U_{k, 2 k-1-2 j}, X\right],\left[X, U_{k, 2 k-2 j}\right]+\left[U_{k, 2 k-1-2 j}, P\right]\right) \\
& =\left(\left[U_{k, 2 k-2-2 j}, P\right]+\left[U_{k, 2 k-3-2 j}, A\right],\left[A, U_{k, 2 k-2 j-2}\right]\right) \\
& =f_{A, P}\left(-U_{k, 2 k-2 j-3}, U_{k, 2 k-2 j-2}\right),
\end{aligned}
$$

i.e. $f_{A, P}\left(\mathbb{Q}_{j+1}\right) \subseteq \operatorname{ad}_{(X, P)}\left(\mathbb{Q}_{j}\right)=\mathfrak{V}_{j} \cdot f_{A, P}$ injective implies $\operatorname{dim}\left(\mathscr{V}_{j}\right)>\operatorname{dim}\left(\mathbb{Q}_{j+1}\right), j=$ $0,1, \ldots, n-1$. Assume from now on that for any $j=1, \ldots, n-1, X^{j} \neq 0$; this condition is generically satisfied. Since $U_{j, 0}=(-1)^{j} X^{j}$ we conclude $\operatorname{ad}_{(X, P)}\left(0, U_{j, 0}\right)$ $=(0,0)$ and hence $\operatorname{dim}\left(\mathscr{Q}_{j}\right) \geqslant 1+\operatorname{dim}\left(\mathfrak{V}_{j}\right)$ for $j \geqslant 1$. Hence we obtain

$$
\operatorname{dim}\left(Q_{j}\right) \geqslant 1+\operatorname{dim}\left(Q_{j+1}\right), \quad j=1, \ldots, n-1, Q_{n}=0
$$

Clearly $\mathbb{Q}_{n-1}=\operatorname{span}\left(0, U_{n-1,0}\right)$ so that $\operatorname{dim}\left(\mathbb{Q}_{n-1}\right)=1$. Repeated application of (4.1) yields then $\operatorname{dim}\left(Q_{1}\right) \geqslant n-1$, which combined with $n-1 \geqslant \operatorname{dim}\left(\mathscr{V}_{0}\right) \geqslant$ $\operatorname{dim}\left(Q_{1}\right)$ gives $\operatorname{dim}(\mathfrak{V})=n-1$. 
TheOrem 4.3. Let $A=\operatorname{diag}\left(a_{1}, \ldots, a_{n}\right)$ have all entries distinct. The $C$. Neumann problem is a completely integrable Hamiltonian system, $n-1$ generically independent integrals in involution being given by

$$
f_{k+1}(X, P)=\frac{1}{2(k+1)} \operatorname{Tr}\left(-\sum_{i=0}^{k} A^{i} X A^{k-i}+\sum_{\substack{i+j+l=k-1 \\ i, j, l>0}} A^{i} P A^{j} P A^{\prime}\right) .
$$

Remarks. (1) The geodesic spray on an ellipsoid in $\mathbf{R}^{n}$ with all axes distinct is also completely integrable and given by the Euler-Poisson equations on the same orbit

$$
\dot{X}=[Q, X], \quad \dot{P}=[Q, P]+\left[X, A^{-1}\right],
$$

for $Q_{i j}=-P_{i j} / a_{i} a_{j}$, with Hamiltonian $E(X, P)=-\frac{1}{2} \kappa(P, Q)+\kappa\left(X, A^{-1}\right)$. It has the same integrals $f_{k}$ since the previous equations can be written as

$$
\left(-X+P \lambda+A \lambda^{2}\right)^{\cdot}=\left[-X+P \lambda+A \lambda^{2},-Q-A^{-1} \lambda\right] .
$$

This follows easily from the work of Moser [10], [11] and has been independently observed by Adler and van Moerbeke [2] who also linearize the flow.

(2) The geodesic spray on $S^{n-1}$ corresponds to $A=0$ in the C. Neumann problem, or to $A=\mathrm{Id}$ in the ellipsoidal problem. The Euler-Poisson equations on the same orbit are $\dot{X}=[P, X], \dot{P}=0$ and the integrals in involution are

$$
f_{k}(X, P)=\left\{\begin{array}{l}
\kappa\left(P^{k}-\left(\frac{1}{n} \operatorname{Tr} P^{k}\right) \mathrm{Id}, X\right), \quad k=\text { even, } \\
\frac{1}{2(k+1)} \operatorname{Tr}\left(P^{k+1}\right), \quad k=\text { odd. }
\end{array}\right.
$$

The Hamiltonian is $-f_{1}(X, P)=-\frac{1}{2} \kappa(P, P)$. The prior proof of independence can be easily modified step-by-step to show that $X_{f_{k}}, k=1, \ldots, n-1$, are generically independent.

ACKNOWLEDGEMENTS. I want to thank $\mathrm{K}$. Uhlenbeck for telling me equations (1.3) which started the present investigation. A conversation with C. Moore on semidirect products is gratefully acknowledged.

\section{BIBLIOGRAPHY}

1. R. Abraham and J. Marsden, Foundations of mechanics, 2nd ed., Benjamin, New York, 1978.

2. M. Adler and P. van Moerbeke, Completely integrable systems, Kac-Moody Lie algebras, and curves and Linearization of Hamiltonian systems, Jacobi varieties, and representation theory, Adv. in Math. (to appear).

3. R. Devaney, Transversal homoclinic orbits in an integrable system, preprint, 1978.

4. V. Guillemin and S. Sternberg, The moment map and collective motion, Ann. of Physics (to appear).

5. A. Iacob and S. Sternberg, Coadjoint structures, solitons, and integrability, Lecture Notes in Phys., vol. 120, Springer, Berlin and New York, 1980.

6. D. Kazhdan, B. Kostant and S. Sternberg, Hamiltonian group actions and dynamical systems of Calogero type, Comm. Pure Appl. Math. 31 (1978), 481-508.

7. B. Kostant; The solution to a generalized Toda lattice and representation theory, M.I.T., 1979, (preprint).

8. H. McKean, Integrable systems and algebraic curves, Lecture Notes in Math., vol. 755, SpringerVerlag, Berlin and New York, 1979. 
9. A. S. Mishchenko and A. T. Fomenko, Euler equations on finite dimensional Lie groups, Math. USSR-Izv. 12 (1978), 371-389.

10. J. Moser, Various aspects of integrable Hamiltonian systems, Dynamical Systems (C.I.M.E., Bressanone, Italy, July 1978), Progress in Math., no. 8, Birkhäuser, Basel.

11. _ Geometry of quadrics and spectral theory, Chern Symposium, Berkeley, 1979 (to appear).

12. C. Neumann, De problemate quodam mechanica, quod ad primam integralium ultra-ellipticorum classem revocatur, J. Reine Angew. Math. 56 (1859), 54-66.

13. T. Ratiu, The motion of the free $N$-dimensional rigid body, Indiana J. Math. 29 (1980), 609-629.

14. __ Involution theorems, Lectures Notes in Math., vol. 755, Springer-Verlag, Berlin and New York, 1980, pp. 219-257.

15. __ Euler-Poisson equations on Lie algebras, Thesis, Univ. of California, Berkeley, 1980.

16. K. Uhlenbeck, Minimal 2-spheres and tori in $S^{k}$, informal preprint, 1975.

17. P. van Moerbeke and T. Ratiu, The Lagrange top (to appear).

Department of Mathematics, University of Michigan, Ann Arbor, Michigan 48109 